\title{
AN INTERACTING PARTICLE PROCESS RELATED TO YOUNG TABLEAUX
}

\author{
ALEXEI BORODIN AND GRIGORI OLSHANSKI
}

\begin{abstract}
We discuss a stochastic particle system consisting of a two-dimensional array of particles living in one space dimension. The stochastic evolution bears a certain similarity to Hammersley's process, and the particle interaction is governed by combinatorics of the Young tableaux.
\end{abstract}

\section{Contents}

1. Introduction 1

2. Generalized standard Young tableaux 2

3. Markov dynamics on generalized tableaux 3

4. Evolution of Gibbs measures

5. Dynamics on infinite generalized tableaux

6. Concluding remarks 8

7. Acknowledgements 9

References 9

\section{INTRODUCTION}

The present note is related to our papers [4], [5], [6], and may be viewed as a companion to the latter paper.

The purpose of [4] and [6] was to construct new models of infinite-dimensional Markov dynamics of representation-theoretic origin.

In [1], we studied a four-parameter family of continuous time Feller Markov processes whose state space is the dual object $\widehat{U(\infty)}$ to the infinite-dimensional unitary group $U(\infty)$. The points of the space $\widehat{U(\infty)}$ parameterize the extremal characters of $U(\infty)$. Equivalently, the same space can also be viewed as the boundary $\partial \mathbb{G T}$ of the Gelfand-Tsetlin graph.

In [6], we described a similar construction related to the infinite symmetric group $S(\infty)$ that leads to a two-parameter family of Feller Markov processes on an infinitedimensional cone $\widetilde{\Omega} \subset \mathbb{R}^{\infty} \times \mathbb{R}^{\infty} \times \mathbb{R}$, called the Thoma cone. The latter space is an amplified version of the dual object to $S(\infty)$ : the base of the Thoma cone is the Thoma simplex $\Omega=\widehat{S(\infty)}$. 
The paper [5] explains a striking resemblance between the character theory of the two groups, $U(\infty)$ and $S(\infty)$ : we show how the characters of the latter group arise as a degeneration of those of the former group. In particular, we explain in what sense the Thoma cone can be viewed as a degeneration of $\partial \mathbb{G} \mathbb{T}$. Moreover, the Markov dynamics on the Thoma cone can also be viewed as a degeneration of the Markov dynamics on $\partial \mathbb{G} \mathbb{T}$.

The Markov processes from [4] and [6] may be viewed as interacting particle processes with nonlocal (or long-range) interaction. On the other hand, as shown in [4], each of the Markov processes on $\partial \mathbb{G} \mathbb{T}$ is "covered" by a certain Markov process with local interaction, living on the path space of the graph $\mathbb{G} \mathbb{T}$. In the present informal note we speculate that similar covering processes should exist in the case of the Markov dynamics on the Thoma cone, too.

Although we do not have a complete proof yet, we could not resist the temptation to introduce this model, because it provides an alternative approach to the results of [6].

The note contains no proofs and presents a collection of conjectures and claims based on some heuristic arguments.

\section{Generalized standard Young tableaux}

Denote $\mathbb{N}:=\{1,2, \ldots\}$ and $\mathbb{N}^{2}:=\mathbb{N} \times \mathbb{N}$. We endow $\mathbb{N}^{2}$ with the structure of poset such that one element $(i, j)$ of $\mathbb{N}^{2}$ is declared to be greater than another element $\left(i^{\prime}, j^{\prime}\right)$ if $i \geq i^{\prime}, j \geq j^{\prime}$, and at least one of these inequalities is strict. By a (finite or infinite) Young diagram we mean a (finite or infinite) subset of $\mathbb{N}^{2}$ such that if it contains a given element of the poset $\mathbb{N}^{2}$ then it also contains all smaller elements.

Let $\mathbb{Y}$ denote the set of all finite Young diagrams including the empty one. We denote a Young diagram by the letter $\lambda$ and write $|\lambda|$ for the size of $\lambda$. (Infinite Young diagram will appear later on.)

Recall that a standard tableau $\tau$ of a given shape $\lambda \in \mathbb{Y}$ is a bijection $\tau: \lambda \rightarrow$ $\{1,2, \ldots,|\lambda|\}$ compatible with the partial order on $\lambda$ inherited from $\mathbb{N}^{2}$. That is, $\tau(i, j)>\tau\left(i^{\prime}, j^{\prime}\right)$ whenever $(i, j)$ is greater than $\left(i^{\prime}, j^{\prime}\right)$.

Let $\mathbb{R}_{>0}$ stand for the set of strictly positive real numbers.

Definition 2.1. Following [5], we define a generalized standard Young tableau of a given shape $\lambda \in \mathbb{Y}$ as a function $h: \lambda \rightarrow \mathbb{R}_{>0}$ such that

- $h$ is compatible with the partial order on $\lambda$, that is, $h(i, j)>h\left(i^{\prime}, j^{\prime}\right)$ whenever $(i, j)$ is greater than $\left(i^{\prime}, j^{\prime}\right)$,

- the numbers $h(i, j)$ are pairwise distinct.

The conventional standard tableaux of a shape $\lambda \in \mathbb{Y}$ encode all possible ways of building $\lambda$ step by step, starting with the empty set and adding an element $(i, j)$ at each step. Likewise, a generalized standard diagram encodes a similar process, only we imagine that elements are added at some moments $h_{1}<h_{2}<\cdots<h_{|\lambda|}$ on the time axis $\mathbb{R}_{>0}$. 
Definition 2.2. Given $r \in \mathbb{R}_{>0}$ and $\lambda \in \mathbb{Y}$, we denote by $H_{r}(\lambda)$ the set of all generalized standard tableaux $h$ of the shape $\lambda$, such that $h(i, j)<r$ for all $(i, j) \in \lambda$. We agree that for the empty diagram $\varnothing$, the set $H_{r}(\varnothing)$ is a singleton. Next, we set

$$
H_{r}:=\bigsqcup_{\lambda \in \mathbb{Y}} H_{r}(\lambda)
$$

Given $h \in H_{r}$, we write $\operatorname{sh}(h)$ for the corresponding Young diagram $\lambda$. It is convenient to define the "height" $h(i, j)$ outside $\lambda$, by setting $h(i, j)=r$ for all $(i, j) \in \mathbb{N}^{2} \backslash \lambda$. Then we may interpret the elements $h \in H_{r}$ as the "height functions" $h: \mathbb{N}^{2} \rightarrow(0, r]$ subject to the following conditions:

- $h(i, j)<h(i, j+1)$ unless $h(i, j)=h(i, j+1)=r$,

- likewise, $h(i, j)<h(i+1, j)$ unless $h(i, j)=h(i+1, j)=r$,

- the set $\operatorname{sh}(h):=\left\{(i, j) \in \mathbb{N}^{2}: h(i, j)<r\right\}$ is finite,

- the numbers $h(i, j)$, where $(i, j)$ ranges over $\operatorname{sh}(h)$, are pairwise distinct.

\section{MARKOV DYNAMiCS ON GENERALIZED TABLEAUX}

As above, we fix a number $r \in \mathbb{R}$. We are going to define a continuous time Markov process on $H_{r}$. It depends on two continuous parameters $z$ and $z^{\prime}$ subject to the following condition:

Condition 3.1. Either both parameters $z$ and $z^{\prime}$ are complex numbers with nonzero imaginary part and $z^{\prime}=\bar{z}$, or both parameters are real and contained in an open unit interval of the form $(m, m+1)$ for some $m \in \mathbb{Z}$.

This is equivalent to requiring that $(z+k)\left(z^{\prime}+k\right)>0$ for every $k \in \mathbb{Z}$. In particular, Condition 3.1 implies that

$$
q(i, j):=(z+j-i)\left(z^{\prime}+j-i\right)>0, \quad \forall(i, j) \in \mathbb{N}^{2},
$$

which is used in the sequel.

We need one more notation. Given $h \in H_{r}$ and $(i, j) \in \mathbb{N}^{2}$, we set

$$
h^{\downarrow}(i, j)= \begin{cases}\max \{h(i-1, j), h(i, j-1)\}, & \text { if } i>1 \text { and } j>1 ; \\ h(1, j-1), & \text { if } i=1, j>1 ; \\ h(i-1,1), & \text { if } i>1, j=1 ; \\ 0, & \text { if } i=j=1 .\end{cases}
$$

Let us fix a couple $\left(z, z^{\prime}\right)$ of parameters satisfying Condition 3.1. We consider an infinite collection of independent Poisson processes indexed by the elements $(i, j) \in$ $\mathbb{N}^{2}$, where each process is defined in the horizontal strip

$$
D_{r}:=\left\{(t, x) \in \mathbb{R}^{2}, \quad-\infty<t<+\infty, \quad 0<x<r\right\}
$$


and the $(i, j)$ th process has constant rate equal to $q(i, j)$ (see (3.1)). We denote by $\pi_{r}(i, j) \subset D_{r}$ the random point configuration from the $(i, j)$ th process. We may assume that $\pi_{r}(i, j)$ has at most one point on each vertical line.

Definition 3.2. We introduce a Markov process $\widetilde{X}_{r}^{\left(z, z^{\prime}\right)}$ on $H_{r}$, whose time evolution is composed of two components, one deterministic and the other one stochastic:

- The deterministic component prescribes the height $h(i, j)$ (for every $(i, j) \in$ $\operatorname{sh}(h))$ to grow with the varying velocity $v(y):=y(y+1)$ depending on the current value $y:=h(i, j)$, until the height attains the maximum value $r$, where it stabilizes.

- The stochastic component, on the contrary, forces the height to instantly drop to some level. More precisely, given $(i, j) \in \mathbb{N}^{2}$, the height $h(i, j)$ drops precisely at those time moments $t$ for which the vertical line with abscissa $t$ contains a point $(t, x) \in \pi_{r}(i, j) \subset D_{r}$ such that the ordinate $x$ satisfies the constraints $h^{\downarrow}(i, j)<x<h(i, j)$. Then the height instantly takes the new value equal to $x$.

Note that the deterministic evolution preserves the set $H_{r}$ : this follows from the very definition of $H_{r}$ and the fact that the velocity $v(x)$ is a strictly increasing function in $x$. Likewise, the stochastic evolution also preserves $H_{r}$, by virtue of the requirement that for the drop to take place $x$ must be greater than $h^{\downarrow}(i, j)$. More pedantically, we had to include in the above definition the additional requirement that a jump $h(i, j) \rightarrow x$ is forbidden whenever $x$ is equal to $h\left(i^{\prime}, j^{\prime}\right)$ for some $\left(i^{\prime}, j^{\prime}\right) \in$ $\operatorname{sh}(h)$ distinct from $(i, j)$, but such an event has probability 0 .

We may interpret $\widetilde{X}_{r}^{\left(z, z^{\prime}\right)}$ as an evolution of a system of particles on $(0, r]$ indexed by the two-dimensional array $\mathbb{N}^{2}$. It is worth noting that the stochastic component of the process includes an interaction between the particles, because the lower bound $h^{\downarrow}(i, j)$ for the possible drop of the $(i, j)$ th particle depends on the positions of the neighboring particles. Here the "neighbors" are defined as the particles with neighboring indices $(i-1, j)$ and $(i, j-1)$. This kind of interaction is similar to that encountered in the exclusion processes.

On the other hand, because of the presence of two components, deterministic and stochastic, the process $\widetilde{X}_{r}^{\left(z, z^{\prime}\right)}$ may be viewed as a member of the class of piecewise deterministic Markov processes introduced by Davis [7].

The following claim looks very plausible to us.

Conjecture 3.3. The process $\widetilde{X}_{r}^{\left(z, z^{\prime}\right)}$ on $H_{r}$ does not explode and so has infinite life time almost surely.

\section{Evolution of GibBs measures}

Here we discuss a connection between the process $\widetilde{X}_{r}^{\left(z, z^{\prime}\right)}$ just defined and the process $X_{r}^{\left(z, z^{\prime}\right)}$ discussed in our papers [6] and [3]. 
Let us recall the definition of $X_{r}^{\left(z, z^{\prime}\right)}$ (see [6, Definition 8.9]). This is a continuous time jump Markov process with the state space $\mathbb{Y}$. The evolution is given the following $Q$-matrix (the matrix of jump rates):

$$
\begin{aligned}
Q_{r}^{\left(z, z^{\prime}\right)}(\lambda, \lambda+\square) & =r(z+c(\square))\left(z^{\prime}+c(\square)\right) \frac{\operatorname{dim}(\lambda+\square)}{(|\lambda|+1) \operatorname{dim} \lambda}, \quad \square \in \lambda^{+}, \\
Q_{r}^{\left(z, z^{\prime}\right)}(\lambda, \lambda-\square) & =(r+1) \frac{|\lambda| \operatorname{dim}(\lambda-\square)}{\operatorname{dim} \lambda}, \quad \square \in \lambda^{-}, \\
-Q_{r}^{\left(z, z^{\prime}\right)}(\lambda, \lambda) & =(2 r+1)|\lambda|+r z z^{\prime} .
\end{aligned}
$$

The notation is the following: $\lambda$ is a Young diagram; $\lambda \pm \square$ is another diagram obtained from $\lambda$ by appending/removing a box $\square ; \lambda^{ \pm}$is the set of those boxes that can be appended to (respectively, removed from) $\lambda$; $Q(\lambda, \lambda \pm \square$ ) is the rate of the jump $\lambda \rightarrow \lambda \pm \square$; finally, $-Q(\lambda, \lambda)$ is equal to the sum of the rates of all possible jumps $\lambda \rightarrow \lambda \pm \square$.

As shown in [6], this $Q$-matrix is regular, meaning that the corresponding jump Markov process does not explode.

Let us return to the sets $H_{r}(\lambda)$ introduced in Definition 2.2. For every $\lambda \in \mathbb{Y}$, let $\operatorname{Tab}(\lambda)$ denote the finite set consisting of all (conventional) standard tableaux of the shape $\lambda$. As seen from Definition 3.2, there is a natural projection $H_{r}(\lambda) \rightarrow \operatorname{Tab}(\lambda)$. For every standard tableau $\tau \in \operatorname{Tab}(\lambda)$, the fiber of this projection over $\tau$ can be viewed as an open simplex $\Delta(\tau)$ of dimension $N=|\lambda|$, formed by the ordered $N$ tuples of reals $\left(x_{1}, \ldots, x_{N}\right)$ such that $0<x_{1}<\cdots<x_{N}<r$. It follows that $H_{r}(\lambda)$ can be viewed as a bounded open subset of $\mathbb{R}^{N}$ whose closure $\overline{H_{r}(\lambda)}$ is a convex polytope endowed with a triangulation.

For instance, if $\lambda=(2,1)$, then $N=3, H_{r}(\lambda)$ consists of the triples $\left(x_{1}, x_{2}, x_{3}\right)=$ $(h(1,1), h(1,2), h(2,1))$ subject to the conditions

$$
0<x_{1}<r, \quad 0<x_{2}<r, \quad 0<x_{3}<r, \quad x_{1}<x_{2}, \quad x_{1}<x_{3}, \quad x_{2} \neq x_{3},
$$

and $\overline{H_{r}(\lambda)}$ is obtained by removing the last inequality and making the remaining inequalities weak. The polytope $\overline{H_{r}(\lambda)}$ is the union of two closed simplices, which are singled out by the inequalities $x_{2} \leq x_{3}$ and $x_{2} \geq x_{3}$, respectively.

Definition 4.1. Recall (see Definition 2.2) that $H_{r}$ is the disjoint union of the sets $H_{r}(\lambda)$, where $\lambda$ ranges over $\mathbb{Y}$. Following [6] we define a Gibbs measure on $H_{r}$ as a probability measure such that its restriction to each subset $H_{r}(\lambda)$ is proportional to the Lebesgue measure. The set of all Gibbs measures is denoted by $\mathscr{G}_{r}$.

Obviously, the natural projection $H_{r} \rightarrow \mathbb{Y}$ establishes a one-to-one correspondence between $\mathscr{G}_{r}$ and the set $\mathscr{M}(\mathbb{Y})$ of all probability measures on $\mathbb{Y}$.

Claim 4.2. The process $\widetilde{X}_{r}^{\left(z, z^{\prime}\right)}$ preserves the set $\mathscr{G}_{r}$ of Gibbs measures, and the evolution of the Gibbs measures induced by the process $\widetilde{X}_{r}^{\left(z, z^{\prime}\right)}$ coincides, under the 
bijection $\mathscr{G}_{r} \rightarrow \mathscr{M}(\mathbb{Y})$, with the evolution of the probability measures on $\mathbb{Y}$ induced by the process $X_{r}^{\left(z, z^{\prime}\right)}$.

Actually, we can rigorously prove only the infinitesimal version of the claim: the application of the infinitesimal generator of $\widetilde{X}_{r}^{\left(z, z^{\prime}\right)}$ to a Gibbs measure translates to the application of the generator of $X_{r}^{\left(z, z^{\prime}\right)}$ to the corresponding measure on $\mathbb{Y}$.

Remark 4.3. Here is a simple yet curious formal identity used in the proof. Assume we are given a standard tableau of the shape $\mathbb{N}^{2}$, that is, a total order on the set $\mathbb{N}^{2}$ compatible with its partial order. Next, for every $(i, j) \in \mathbb{N}^{2}$, set

$$
(i, j)^{\downarrow}:= \begin{cases}\max \{(i-1, j),(i, j-1)\}, & \text { if } i>1 \text { and } j>1, \\ (1, j-1), & \text { if } i=1, j>1, \\ (i-1,1), & \text { if } i>1, j=1, \\ \text { undefined, } & \text { if }(i, j)=(1,1),\end{cases}
$$

and

$$
(i, j)^{\uparrow}:=\min \{(i+1, j),(i, j+1)\},
$$

where the maximum and minimum are taken relative to the prescribed total order on $\mathbb{N}^{2}$. Finally, attach to every $(i, j) \in \mathbb{N}^{2}$ a formal variable $y(i, j)$. Then the identity in question is

$$
\sum_{(i, j) \in \mathbb{N}^{2}}\left(y\left((i, j)^{\uparrow}\right)+y\left((i, j)^{\downarrow}\right)-2 y(i, j)\right)(z+j-i)\left(z^{\prime}+j-i\right)=2 \sum_{(i, j) \in \mathbb{N}^{2}} y(i, j)
$$

with the agreement that $y\left((1,1)^{\downarrow}\right):=0$.

As explained in [6], the process $X_{r}^{\left(z, z^{\prime}\right)}$ has a stationary distribution, the so called mixed z-measure, denoted by $M_{r}^{\left(z, z^{\prime}\right)}$. Let $\widetilde{M}_{r}^{\left(z, z^{\prime}\right)}$ denote the corresponding Gibbs measure on $H_{r}$. Claim 4.2 implies that $\widetilde{M}_{r}^{\left(z, z^{\prime}\right)}$ serves as the stationary distribution for the process $\widetilde{X}_{r}^{\left(z, z^{\prime}\right)}$.

Claim 4.2 says that the process $\widetilde{X}_{r}^{\left(z, z^{\prime}\right)}$ in some sense "covers" the jump process $X_{r}^{\left(z, z^{\prime}\right)}$. Note that Young diagrams $\lambda \in \mathbb{Y}$ can be represented as particle configurations, so that $X_{r}^{\left(z, z^{\prime}\right)}$, like $\widetilde{X}_{r}^{\left(z, z^{\prime}\right)}$, can also be interpreted as an interacting particle process. However, a substantial difference between $\widetilde{X}_{r}^{\left(z, z^{\prime}\right)}$ and $X_{r}^{\left(z, z^{\prime}\right)}$ is that the particle interaction is local in the former process and highly non-local in the latter one. In this sense, $\widetilde{X}_{r}^{\left(z, z^{\prime}\right)}$ seems to be simpler than $X_{r}^{\left(z, z^{\prime}\right)}$.

We showed in [6] that for every pair $r^{\prime}>r$ there exists a "link" $\Lambda_{r}^{r^{\prime}}$ (an infinite stochastic matrix of format $\mathbb{Y} \times \mathbb{Y}$ ), which intertwines the processes $X_{r^{\prime}}^{\left(z, z^{\prime}\right)}$ and $X_{r}^{\left(z, z^{\prime}\right)}$ for all $\left(z, z^{\prime}\right)$. For the covering processes the picture is simpler in the sense that the processes $\widetilde{X}_{r^{\prime}}^{\left(z, z^{\prime}\right)}$ and $X_{r}^{\left(z, z^{\prime}\right)}$ are "linked" by an ordinary map, the truncation map (5.1) that we now define. 


\section{Dynamics on infinite Generalized tableAuX}

Given $r^{\prime}>r$, we define the truncation map $H_{r^{\prime}} \rightarrow H_{r}$ as the transform

$$
h(i, j) \rightarrow \min (h(i, j), r), \quad \forall(i, j) \in \mathbb{N}^{2} .
$$

Obviously, for a triple $r^{\prime \prime}>r^{\prime}>r$, the composed map $H_{r^{\prime \prime}} \rightarrow H_{r^{\prime}} \rightarrow H_{r}$ is the same as $H_{r^{\prime \prime}} \rightarrow H_{r}$. Therefore, we may define the projection limit space

$$
H:=\lim _{\longleftarrow} H_{r}, \quad r \rightarrow+\infty .
$$

The elements of $H$ can be viewed as the functions $h: \mathbb{N}^{2} \rightarrow \mathbb{R}_{>0} \cup\{+\infty\}$ such that

- $h(i, j)<h(i+1, j)$ unless $h(i, j)=h(i+1, j)=+\infty$;

- $h(i, j)<h(i, j+1)$ unless $h(i, j)=h(i, j+1)=+\infty$;

- the finite values $h(i, j)$ are pairwise distinct and do not have accumulation points on $\mathbb{R}_{>0}$.

Note that the natural projection $H \rightarrow H_{r}$ is still given by (5.1). Note also that, for $h \in H$, the set

$$
\operatorname{sh}(h):=\left\{(i, j) \in \mathbb{N}^{2}: h(i, j)<+\infty\right\} \subseteq \mathbb{N}^{2}
$$

is a (possibly infinite) Young diagram.

Every element $h \in H$ may be interpreted as a system of particles on the extended halfline $\mathbb{R}_{>0} \cup\{+\infty\}$, indexed by $\mathbb{N}^{2}$ (we assume that the $(i, j)$ th particle has coordinate $x=h(i, j))$. If $\operatorname{sh}(h)=\mathbb{N}^{2}$, then all particles are on $\mathbb{R}_{>0}$, but it may happen that $\operatorname{sh}(h)$ is a proper subset of $\mathbb{N}^{2}$; then there is an infinite reservoir of particles at infinity, indexed by the elements of $\mathbb{N}^{2} \backslash \operatorname{sh}(h)$. An important requirement included in the definition is that the particle configuration on $\mathbb{R}_{>0}$ is always locally finite meaning that there are finitely many particles in any bounded interval.

Claim 5.1. The family of Markov processes $\left\{\widetilde{X}_{r}^{\left(z, z^{\prime}\right)}: r \in \mathbb{R}_{>0}\right\}$ is consistent with the truncation maps $H_{r^{\prime}} \rightarrow H_{r}$ and so there exists a unique Markov process $\widetilde{X}^{\left(z, z^{\prime}\right)}$ on the space $H$, consistent with this family by means of the truncation maps $H \rightarrow H_{r}$.

In other words, $\widetilde{X}^{\left(z, z^{\prime}\right)}$ can be viewed as the projective limit of the processes $\widetilde{X}_{r}^{\left(z, z^{\prime}\right)}$.

The Markov dynamics of such a system, given by the process $\widetilde{X}^{\left(z, z^{\prime}\right)}$, is described in the same way as for the truncated processes $\widetilde{X}_{r}^{\left(z, z^{\prime}\right)}$, see Section 3 above, only the open interval $(0, r)$ should be replaced by the halfline $\mathbb{R}_{>0}$, and the endpoint $r$ is shifted to $+\infty$.

Informally, in the particle system interpretation, the deterministic component looks as the accelerating movement of particles to the right with velocity $v(y)=y(y+$ 1 ), while the the concurrent stochastic component forces the particles to instantly jump to the left. The latter component is driven by a two-dimensional array $\{\pi(i, j)$ : $\left.(i, j) \in \mathbb{N}^{2}\right\}$ of independent Poisson processes in the half-plane $D:=\{(t, x): t \in$ $\mathbb{R}, x>0\}$, where the $(i, j)$ th process has constant rate $q(i, j)$ given by (3.1). Note that the particles can escape from $\mathbb{R}_{>0}$ to infinity and return back to $\mathbb{R}_{>0}$. 
Note also that the sample trajectories of $\widetilde{X}^{\left(z, z^{\prime}\right)}$ look more sophisticated than those of the truncated process $\widetilde{X}_{r}^{\left(z, z^{\prime}\right)}$. This is due to a major difference between the Poisson processes in the strip $D_{r}$ and in the half-plane $D$ : the pushforward of a Poisson configuration under the projection to the $t$-axis is locally finite in the former case but not in the latter case.

Now we extend our definition of Gibbs measures to the space $H$.

Definition 5.2. A probability measure on $H$ is said to be a Gibbs measure if its pushforward under the truncation map $H \rightarrow H_{r}$ is a Gibbs measure on $H_{r}$ in the sense of Definition 4.1, for every $r \in \mathbb{R}_{>0}$. The set of all Gibbs measures on $H$ is denoted by $\mathscr{G}$.

The above discussion shows that the process $\widetilde{X}^{\infty}$ preserves the Gibbs measures.

On the other hand, as explained in [5], there is a one-to-one correspondence $\mathscr{G} \leftrightarrow \mathscr{M}(\widetilde{\Omega})$, where $\widetilde{\Omega}$ is an infinite-dimensional cone in $\mathbb{R}^{\infty} \times \mathbb{R}^{\infty} \times \mathbb{R}$, called the Thoma cone, and $\mathscr{M}(\widetilde{\Omega})$ denotes the space of probability measures on $\widetilde{\Omega}$. Further, we showed in [6] that the family $\left\{X_{r}^{\left(z, z^{\prime}\right)}: r \in \mathbb{R}_{>0}\right\}$ of Markov processes on $\mathbb{Y}$ determines, via the links $\Lambda_{r}^{r^{\prime}}$, a Markov process $X^{\left(z, z^{\prime}\right)}$ on the Thoma cone $\widetilde{\Omega}$. Because of the bijection $\mathscr{G} \leftrightarrow \mathscr{M}(\widetilde{\Omega})$, this leads to the following conclusion:

Claim 5.3. The process $\widetilde{X}^{\left(z, z^{\prime}\right)}$ covers the process $X^{\left(z, z^{\prime}\right)}$ in the sense that the evolution of arbitrary probability measures on the Thoma cone induced by $X^{\left(z, z^{\prime}\right)}$ is the same as the evolution of the Gibbs measures on $H$ induced by $\widetilde{X}^{\left(z, z^{\prime}\right)}$.

Here is a reformulation. Given a point $\omega \in \widetilde{\Omega}$, let us denote by $G_{\omega}$ the Gibbs measure on $H$ corresponding to the Dirac measure at $\omega$. The correspondence $\omega \mapsto$ $G_{\omega}$ can be viewed as a Markov kernel $\Lambda_{H}^{\widetilde{\Omega}}=\Lambda_{H}^{\widetilde{\Omega}}(\omega, d h)$. Then the above claim means that $\Lambda_{H}^{\widetilde{\Omega}}$ intertwines $X^{\left(z, z^{\prime}\right)}$ and $\widetilde{X}^{\left(z, z^{\prime}\right)}$.

In [6], we showed that the process $X^{\left(z, z^{\prime}\right)}$ on the Thoma cone has a stationary distribution $M^{\left(z, z^{\prime}\right)}$, which is defined by the family $\left\{M_{r}^{\left(z, z^{\prime}\right)}: r \in \mathbb{R}_{>0}\right\}$ via the links $\Lambda_{r}^{r^{\prime}}$. Let $G^{\left(z, z^{\prime}\right)}$ stand for the corresponding Gibbs measure on $H$; in other words, $G^{\left(z, z^{\prime}\right)}$ is the transform of $M^{\left(z, z^{\prime}\right)}$ by $\Lambda_{H}^{\widetilde{\Omega}}$.

Claim 5.4. The Gibbs measure $G^{\left(z, z^{\prime}\right)}$ serves as a stationary distribution for $\widetilde{X}^{\left(z, z^{\prime}\right)}$.

\section{Concluding REMARKS}

Besides the truncation maps $H \rightarrow H_{r}$ there exist other projections under which the Markov property of the process $\widetilde{X}^{\left(z, z^{\prime}\right)}$ is not destroyed. Namely, one may fix an arbitrary Young diagram, finite or infinite, and focus on those particles that are indexed by the elements $(i, j)$ of that diagram.

The simplest such example is obtained when the diagram in question consists of the single element $(1,1)$. Then the model represents a single particle that moves on 
the extended halfline $\mathbb{R}_{>0} \cup\{+\infty\}$. The dynamics is described exactly as before: a deterministic movement to the right with the velocity $v(y)=y(y+1)$ combined with instant jumps to the left directed by the Poisson process in the half-plane $D$ with constant rate $q(1,1)=z z^{\prime}$.

A more complicated model arises when we take the infinite one-row diagram $\{(1, j): j \in \mathbb{N}\}$. Then we have countably many particles, enumerated by the numbers $j \in \mathbb{N}$ and occupying positions $x_{1}<x_{2}<\ldots$ on $\mathbb{R}_{>0}$ with a possible infinite reservoir at infinity, meaning that it may happen that $x_{j}=+\infty$ for all $j$ large enough. The dynamics in this model has a certain resemblance with the Hammersley process studied in Aldous-Diaconis [1].

Finally, note that the process $\widetilde{X}^{\left(z, z^{\prime}\right)}$ admits a Plancherel-type degeneration in the spirit of [6, Section 10]. The idea is to scale the space variable $x$ by substituting $x\left(z z^{\prime}\right)^{-1}$ instead of $x$, and pass to a limit as both $z$ and $z^{\prime}$ go to $+\infty$. Then we get a simplified model, where the velocity in the deterministic component depends on the coordinate $y \in \mathbb{R}_{>0}$ via $v(y)=y$, and the backward jump rules are the same for all particles (they are governed by independent Poisson processes in $D$ with constant rate $q=1)$.

\section{AcKnowledgements}

A. B. was partially supported by NSF-grant DMS-1056390. G. O. was partially supported by a grant from Simons Foundation (Simons-IUM Fellowship), the RFBR-CNRS grant 10-01-93114, and the project SFB 701 of Bielefeld University.

\section{REFERENCES}

[1] D. Aldous and P. Diaconis, Hammersley's interacting particle process and longest increasing subsequences. Prob. Theory Rel. Fields 103, 199-213.

[2] A. Borodin and G. Olshanski, Stochastic dynamics related to Plancherel measure on partitions. In: Representation Theory, Dynamical Systems, and Asymptotic Combinatorics (V. Kaimanovich and A. Lodkin, eds). Amer. Math. Soc. Translations - Series 2, vol. 217, 2006, 9-22; arXiv:math-ph/0402064.

[3] A. Borodin and G. Olshanski, Markov processes on partitions. Probab. Theory Rel. Fields 135 (2006), 84-152; arXiv:math-ph/0409075.

[4] A. Borodin and G. Olshanski, Markov processes on the path space of the Gelfand-Tsetlin graph and on its boundary. J. Funct. Anal. 263 (2012), no. 1, 248-303; arXiv:1009.2029.

[5] A. Borodin and G. Olshanski, The Young bouquet and its boundary. Moscow Math. J. 13 (2013), no. 2; arXiv:1110.4458.

[6] A. Borodin and G. Olshanski, Markov dynamics on the Thoma cone: a model of time-dependent determinantal processes with infinitely many particles. Preprint, arXiv:1303.????

[7] M. H. A. Davis, Piecewise-deterministic Markov processes: A general class of nondiffusion stochastic models. J. Royal Stat. Soc. Ser. B (Methodological) 46 (1984), 353388. 
Alexei Borodin:

Department of Mathematics, Mit, Cambridge, MA, USA;

Institute for Information Transmission Problems, Moscow, Russia

E-mail address: borodin@math.mit.edu

Grigori Olshanski:

Institute for Information Transmission Problems, Moscow, Russia;

INDEPENDENT UNIVERSITY OF MOSCOW, Russia;

National Research University Higher School of Economics, Moscow, Russia

E-mail address: olsh2007@gmail.com 